Se puede concluir que aunque se piense qua hasta ahora se está aplicando la administración y esta idea en realidad es errónea pues como se ha podido analizar esta viene de mucho antes y sus ideas provienen de raíces históricas que hoy en día se están aplicando con la única diferencia de que se busca el perfeccionamiento al aplicar nuevas tendencias de la administración y los cambios en la vida cotidiana.

\title{
BIBLIOGRAFÍA
}

- CERTO, Samuel. Administración Moderna. Prentice Hall, 2001

- CHIAVENATO, Idalberto. Administración. Mc Graw Hill, 2001.

- RAMIREZ, Carlos. Fundamentos de Administración. Ecoe Ediciones, 1999

- STONER, James. Administration. Prentice Hall, 1996

- SCHERMERHORN, John. Administración. Limusa Wiley, 2003

\section{EN BÚSQUEDA \\ DE LA CIENTIFICIDAD DEL TURISMO}

\section{Bibiela Mojica Tibaduiza}

Paola Carolina Gama

Grupo de Investigación para la Animación Culturạl "Muisuata"

Este artículo es un primer acercamiento al proyecto LA INVESTIGACIÓN EN TURISMO: UNA VISION DESDE LA ESCUELA DE ADMISTRACION TURISTICA Y HOTELERA (1986 - 2008) que se viene desarrollando en torno a la Tradición Investigativa en la Escuela de Administración Turística y Hotelera- EATH de la UPTC evidenciada en los trabajos de grado de los estudiantes que se han realizado desde el año 1986 hasta el 2008. Este documento abordara las siguientes temáticas, importantes para lograr el sentido de la investigación en turismo: el lugar de la investigación, la importancia del turismo, la investigación en turismo y la problemática frente a la realidad de la investigación en turismo contribuyendo a la orientación, apropiación y posibles alternativas de solución. La investigación es un lugar de conocimientos y encontrar el lugar que se merece investigar en investigación se constituye en un aspecto fundamental ya que permite develar que posición adopta la investigación en el espacio como el de la formación, el de la política entre otros. El definir el lugar de la investigación obliga a devolver las preguntas hacia las instituciones y los sujetos que investigan como, desde donde y para que investigan.

La investigación tiene capacidad de alterar lo establecido, mostrar alternativas, dislocar lo visible, revelar situaciones y procesos que estaban a la vista pero 
pasaban inadvertidos. Por tal motivo la función de la investigación es producir conocimiento que permite informar a los que toman las decisiones, o dicho de otro modo aportar evidencias informadas, o como lo opuesto del sentido común contribuye a la reflexión acerca de la investigación que surge de una búsqueda que linda con la angustia. (MESSINA,G; 2008:6-7)

En este sentido el turismo es importante porque según la Organización Mundial del Turismo- OMT citada por Jafari (2005: 1) es la mayor fuerza económica del mundo $y$ un gigante industrial de dimensiones globales, que inicio su crecimiento continuado después de la segunda guerra mundial. Esto evidenciado en la actividad turística que ha traído consigo beneficios que se difunden por toda la sociedad consolidándose como una alternativa económicamente viable para las comunidades de los diferentes países que trabajan en el turismo. De igual manera el turismo ha contribuido a con beneficios Socioculturales como educación, comprensión entre pueblos y la paz, preservación y valoración de la cultura.

La complejidad del turismo conlleva a investigar en esta área y constituye un compromiso por parte de los profesionales para pasar de la concepción que se tiene de lo pragmático a cientificidad del turismo. Promover la investigación en turismo permite la introducción de nuevos conocimientos refutando, consolidando o confirmando o no los ya existentes también logra el aumento de la cantidad y calidad de publicaciones dedicados al turismo, el crecimiento de la formación en turismo por ende el número de escuelas y universidades, conformación de nuevos grupos y asociaciones de estudiosos y profesionales y lo mas importante a ayuda en la toma de decisiones del sector publico y privado ya que de esto depende el desarrollo $y$ proyección de la actividad turística. (REJOWSKI, M: 2009:2)

La problemáticas frente a la realidad de la investigación en turismo desde la perspectiva de Jafari considera que la comunidad investigativa en turismo es pequeña pero creciente y que están formados principalmente en ciencias sociales como la antropología, psicología, sociología, con ello evidenciando la falta de investigación por parte de especialistas en turismo. Desde la percepción de Gómez (2005:3-7)el problema radica en la poca comprensión del turismo y la escasa cientificidad de sus estudios, eEl escaso y pobre conocimiento turístico, la fragmentación del conocimiento turístico y la falta de ética y profesionalismo en la investigación. REJOWSKI, M. (2009:4) enfoca la problemática en la poca integración y relacionamiento entre investigadores, precarios recursos documentales, falta de reconocimiento $y$ apoyo a los investigadores turísticos. Desde nuestra perspectiva en el grupo de investigación para la Animación Cultural "MUISUATA" de la UPTC, las dificultades que afrontamos en torno a la 
investigación en turismo se reflejan en la inexistencia de un balance documental del estado de la investigación de la EATH- UPTC y develar el lugar que esta tiene con respecto a las tendencias mundiales de investigación. Se busca aportar con la Elaboración un libro sobre la Investigación en Turismo que permita reorientar la percepción y formación en Turismo en la EATH, la estructuración de las líneas de investigación y Diseñar e implementar un software optimo para el manejo de los Balances Documentales en Turismo a disposición de los estudiantes, para una mejor comprensión de las investigaciones futuras, a la vez lograr Proponer un diplomado sobre Turismo en la UPTC.

\section{BIBLIOGRAFÍA}

-JAFAR, Jafari (2005). "La cientificación del turismo" en contribuciones a la economía, julio. Texto completo en: http://www.eumed.net/ce/

-GOMEZ NIEVES, Salvador (2005). Repensar en el turismo: ante la irresistijle tentación de lo pragmático y lo empírico. En: VII Congreso Nacional y I Internacional de Investigación en Turismo. Universidad de Guadalajara.

-MESSINA, Graciela. (2008) El lugar de la Investigación en la Construcción de lo Público. -REJOWSKI, M. (2009) "Investigación en Turismo en las Universidades Brasileñas" Universidad de Sao Paulo, Brasil.

\section{SENDEROS INTERPRETATIVOS,}

UNA MIRADA DESDE EL DEPARTAMENTO DE BOYACÁ

Flor Maria Muñoz Vargas

Jenny Alexandra Bautista

Grupo de Investigación para la Animación Cultural "MUISUATA"

La experiencia de atravesar un bosque, salir a un

Páramo luminoso, llegar a una cascada y sentir de cerca el ímpetu del agua es tan creativa que no necesitan comentarios, salvo admiración silencio ò poesía.

Tomás Estevez

Abordar la temática de los senderos interpretativos implica, necesariamente, retomar conceptos como: Turismo, Turismo rural. Ecoturismo y Senderos interpretativos. Así pues, el Turismo se puede definir como "el fenómeno 